\title{
Long-term efficacy and safety of vedolizumab in patients with inflammatory bowel diseases: A real-life experience from a tertiary referral center
}

\author{
Gabriele Dragoni $^{1,2}$ ( ) | Siro Bagnoli ${ }^{1}$ | Marco Le Grazie ${ }^{1}$ | Claudia Campani ${ }^{1}$ | \\ Francesca Rogai $^{1}$ | Natalia Manetti ${ }^{1}$ | Carolina Bensi ${ }^{1}$ | Giuseppe Macri ${ }^{1}$ | \\ Andrea Galli $^{2}$ | Monica Milla ${ }^{1}$
}

${ }^{1}$ IBD Referral Center, Gastroenterology Department, Careggi University Hospital, Florence, Italy

${ }^{2}$ Gastroenterology Research Unit, Department of Experimental and Clinical Biochemical Sciences, Mario Serio University of Florence, Florence, Italy

\section{Correspondence}

Gabriele Dragoni, Department of Gastroenterology, IBD Referral Center, Careggi University Hospital, Viale San Luca, 50134, Florence, Italy.

Email: gabriele.dragoni@unifi.it
Objective: The study aimed to evaluate the long-term efficacy and safety of vedolizumab in a real-life cohort of patients with inflammatory bowel diseases enrolled at a tertiary referral center.

Methods: Data were retrospectively collected from August 2016 to November 2018. The primary outcomes were clinical response and remission at 14, 24, and 52 weeks, and steroid-free remission rate (SFRR) at 52 weeks. Endoscopic response and remission rates at 52 weeks were the secondary outcomes.

Results: Altogether 49 patients (22 with ulcerating colitis [UC] and 27 with Crohn's Disease [CD]) were enrolled. The clinical response rate gradually dropped from $85 \%$ and $50 \%$ in CD and UC, respectively, at week 14 to $59 \%$ and $25 \%$ at week 52, with significantly a higher response in $C D$ at week 14 . The endoscopic response at week 52 was $55 \%$ in CD and $25 \%$ in UC $(P=0.21)$. CD group had a higher SFRR than UC group (41\% vs $20 \%$ ) at 52 weeks, although the difference was not statistically significant. Similar clinical and endoscopic rates were observed in biologic-naive and -experienced patients. We reported no discontinuation due to adverse drug reactions, and only mild to moderate events.

Conclusions: In our cohort the clinical response in the induction phase was similar to those of registered trials, despite surprising better results for $C D$. During the maintenance phase we observed an higher drop out than in the reported literatures. Of note, its good safety profile makes vedolizumab a reliable choice in patients with contraindications to anti-tumor necrosis factor agents.

\section{KEYWORDS}

adverse event, anti-integrin, endoscopic remission, inflammatory bowel diseases, maintenance, swap therapy 


\section{1 | INTRODUCTION}

The control of inflammation in inflammatory bowel diseases (IBD), both in patients with Crohn's disease (CD) and ulcerative colitis (UC), has been a major topic of research in the last 20 years. The development of specific anti-tumor necrosis factor (TNF) agents is considered the first fundamental step in IBD management and this has allowed the achievement of deep remission in a high proportion of patients. ${ }^{1}$ Unfortunately, $10 \%-30 \%$ of patients are primary non-responders to anti-TNF and more than $50 \%$ of responders lose control over time. ${ }^{2}$ Therefore, new therapeutic agents with different targets of inflammation are deeply needed. ${ }^{3}$

Vedolizumab (VDZ) is a fully human immunoglobulin G1 monoclonal antibody selective for the gut. It has specific action against leukocyte gut homing, blocking the integrin $\alpha 4 \beta 7$ on circulating lymphocytes and preventing their diapedesis through the endothelial walls. ${ }^{4}$ Three registered trials (GEMINI) have demonstrated the efficacy and safety of the molecule in patients who are naive or experienced with anti-TNF. ${ }^{5-7}$ Long-term efficacy and safety up to 5 years of follow-up of these cohorts have also been reported. ${ }^{8,9}$

Considering the highly selective character of trial enrollment, it is always fundamental to observe whether real-life settings support the initially reported results of a new drug. ${ }^{10}$ Real-world publications on this field are accumulating, with some conflicting results, ${ }^{11-31}$ but only a few of them include long-term evaluation at 1 year with endoscopic assessment. $^{20,27,31}$ Therefore, we presented an Italian single-center study on VDZ long-standing effectiveness and tolerability in patients with $U C$ and $C D$ at a tertiary referral setting.

\section{2 | PATIENTS AND METHODS}

Upon Institutional Review Board approval, the prospectively maintained biologics database at the IBD Referral Center of the Florence University Hospital was retrospectively analyzed. From August 2016 to November 2018, two gastroenterologists with experiences in the IBD field collected data for all adult ( $\geq 18 \mathrm{y}$ ) patients with IBD who had started treatment with VDZ between July 2016 and July 2018 and who had completed at least the induction regimen. All patients, except one with IBD undefined (IBD-U), had an established diagnosis of UC or $\mathrm{CD}$ according to the European evidence-based guidelines. ${ }^{32,33}$

\section{1 | Medical workup}

Clinical history of all the patients were collected and they underwent physical examination before starting VDZ treatment. Furthermore, routine screening for tuberculosis (both serology and chest radiography), common viruses (Epstein-Barr virus, cytomegalovirus, varicella zoster virus, human immunodeficiency virus, hepatitis $B$ and $C$ viruses) and antinuclear antibodies was carried out, as recommended by the guidelines. ${ }^{34}$

Clinical activity was classified according to the partial Mayo score (PMS) for UC and the Harvey-Bradshaw index (HBI) for CD and recorded at week 0, 14, 24, and 52. ${ }^{35,36}$ Endoscopic assessment was classified according to the Mayo endoscopic subscore (MAYO) for UC, the simple endoscopic score for Crohn's Disease (SES-CD) for non-resected patients with $C D$, while the Rutgeerts score was used for the CD group with prior surgery. ${ }^{37-39}$ These evaluations were performed at baseline and at week 52 for patients with a clinical response, and just before the ending point in patients without clinical improvement. Patients' comorbidities were evaluated with the Charlson comorbidity index $(\mathrm{CCl}){ }^{40}$

After having given their signed informed consents, patients were administered with VDZ 300 mg (Entyvio; Takeda Pharmaceutical, Tokyo, Japan) intravenously at week 0, 2 and 6, and then every 8 weeks. Patients with CD who had no clinical response to the induction regimen received an additional dose at week 10 . Dose intensification every 4 weeks was an option for patients with a partial loss of response. The last follow-up was recorded in November 2018. For patients who withdrew from VDZ, the date of the last follow-up visit was set as the time point of discontinuation. All possible adverse drug reactions were recorded.

\subsection{Study outcomes}

The primary outcomes were clinical response and remission rates at 14, 24, and 52 weeks, and steroid-free remission rate at 52 weeks. A clinical response was defined as a PMS $<4$ or a reduction of at least $30 \%$ of activity for UC and IBD- $\mathrm{U}$, and a $\mathrm{HBI}<7$ or a reduction of at least 3 points for $C D$. Clinical remission was defined as a PMS of $0-1$ for $\mathrm{UC}$ and IBD-U, and an $\mathrm{HBI}<4$ for $\mathrm{CD}$. The steroid-free remission rate was defined as clinical remission with no need for systemic corticosteroids.

The secondary outcomes were endoscopic response and remission rates at 52 weeks, and the safety profile both in monotherapy and in combination with immunosuppressants. Endoscopic response was defined as a MAYO score of 0-1 for UC and IBD-U, and SES-CD or a Rutgeerts score of $<6$ or i0-i1, respectively, for CD. Endoscopic remission was defined as MAYO score of 0 for $U C$ and IBD- $U$, and SES-CD or Rutgeerts score, respectively, of 0-2 or i0 for CD. Among biochemical markers, only $\mathrm{C}$-reactive protein (CRP) was evaluated.

\section{3 | Statistical analysis}

Two major groups of UC (including the patient with IBD-U) and CD cohorts were enrolled for data collection and analysis. We took into account the following variables to test the heterogeneity of the two groups: sex, age at diagnosis and disease duration, age at administration of $\mathrm{VDZ}, \mathrm{CCl}$, disease duration, extra-intestinal manifestations, baseline clinical or endoscopic activity, baseline CRP level, previous anti-TNF exposure, and concomitant combination therapies.

Descriptive data were obtained for the groups reporting median and interquartile range (IQR) for continuous variables, while numbers and frequencies or proportions were used for categorical variables. Comparisons between categorical variables were performed with the $\chi^{2}$ test or Fisher's exact test for small samples; and comparisons 
TABLE 1 Baseline characteristics of patient cohorts

\begin{tabular}{|c|c|c|c|}
\hline $\operatorname{Sex}(n, \%)$ & $U C(n=22)$ & $C D(n=27)$ & $P$ value \\
\hline Male & $9(41)$ & $19(70)$ & 0.07 \\
\hline Female & $13(59)$ & $8(30)$ & \\
\hline Age at diagnosis (y) (median, IQR) & $49.5(29-59.5)$ & $25(16-37)$ & 0.006 \\
\hline Age at VDZ administration (y) (median, IQR) & $58(38-68)$ & $44(30-69)$ & 0.19 \\
\hline Disease duration (y) (median, IQR) & $8(4.5-13.5)$ & $15(8-27)$ & 0.01 \\
\hline \multicolumn{4}{|l|}{ Charlson comorbidity index (n, \%) } \\
\hline 0 & $7(32)$ & $11(41)$ & 0.73 \\
\hline$\geq 1$ & $15(68)$ & $16(59)$ & \\
\hline
\end{tabular}

Montreal classification for $C D(n, \%)$

Age at diagnosis $(\mathrm{y})$

$$
\mathrm{A} 1(\leq 16)
$$

A2 (17-40)

$15(55)$

A3 (>40)

5 (19)

Location

L1 (ileal)

L2 (colonic)

$1(4)$

L3 (ileocolonic)

$18(67)$

L4 (isolated upper disease) ${ }^{\mathrm{a}}$

4 (15)

Behavior

B1 (non-stricturing, non-penetrating)

B2 (stricturing)

$13(48)$

B3 (penetrating)

9 (33)

$\mathrm{P}$ (perianal disease)

10 (37)

Montreal classification for ulcerative colitis ( $n, \%)$
E1 (proctitis)
1 (5)
E2 (distal colitis)
$10(45)$
E3 (pancolitis)
$11(50)$

Extra-intestinal manifestations ( $\mathrm{n}, \%)$

Yes

8 (36)

$14(52)$

0.42

No

$14(64)$

$13(48)$

Basal endoscopic activity (MAYO and SES-CD) (n, \%)

Severe

$17(77)$

$15(55)$

0.2

Non severe

5 (23)

$12(45)$

Basal clinical activity

Severe

$20(91)$

25 (93)

1

Non severe

2 (9)

2 (7)

Basal CRP (ULN $0.5 \mathrm{mg} / \mathrm{dL}$ ) (median, IQR)

$0.52(0.30-1.95)$

$0.8(0.3-2)$

0.5

Corticosteroids during therapy (n, \%)

Yes

$17(77)$

$15(56)$

0.14

No

$5(23)$

$12(44)$

Previous administration of anti-TNF (n, \%)

No

Yes

- 1 Biologic 
TABLE 1 (Continued)

$\begin{array}{lcc}\text { Sex }(\mathrm{n}, \%) & \text { UC }(\mathbf{n}=22) & \mathrm{CD}(\mathrm{n}=27) \\ \text { COMBO (VDZ + IMS) (n, \%) } & & 7(26) \\ \text { Yes } & 9(41) & 20(74)\end{array}$

a $\mathrm{L} 4$ can be added to L1-L3 when concomitant upper gastrointestinal disease is present.

Abbreviations: CD, Crohn's disease; CRP, C-reactive protein; IMS, immunosuppressants; IQR, interquartile range; MAYO, Mayo endoscopic subscore; n.a., not applicable; SES-CD, simple endoscopic score for Crohn's Disease; TNF, tumor necrosis factor; UC, ulcerative colitis; ULN, upper limit of normal; VDZ, vedolizumab.

TAB LE 2 Response and remission rates at 14, 24, and 52 wks (n, \%)

\begin{tabular}{|c|c|c|c|}
\hline & $\begin{array}{l}U C \\
N=22\end{array}$ & $\begin{array}{l}C D \\
N=27\end{array}$ & $\begin{array}{l}P \\
\text { value }\end{array}$ \\
\hline Clinical response at $14 \mathrm{wks}$ & $11 / 22(50)$ & $23 / 27(85)$ & 0.01 \\
\hline Clinical remission at 14 wks & $7 / 22(32)$ & $18 / 27(67)$ & 0.02 \\
\hline Clinical response at $24 \mathrm{wks}$ & $13 / 22(59)$ & $23 / 27(85)$ & 0.055 \\
\hline Clinical remission at 24 wks & $7 / 22(32)$ & $16 / 27(59)$ & 0.04 \\
\hline Clinical response at $52 \mathrm{wks}^{\mathrm{a}}$ & $5 / 20(25)$ & $13 / 22(59)$ & 0.06 \\
\hline Clinical remission at $52 \mathrm{wks}^{\mathrm{a}}$ & $4 / 20(20)$ & $10 / 22(45)$ & 0.11 \\
\hline $\begin{array}{l}\text { Endoscopic response at } \\
52 \mathrm{wks}^{\mathrm{a}}\end{array}$ & $5 / 20(25)$ & $12 / 22(55)$ & 0.21 \\
\hline $\begin{array}{l}\text { Endoscopic remission at } \\
52 \mathrm{wks}^{\mathrm{a}}\end{array}$ & $4 / 20(20)$ & $6 / 22(27)$ & 0.72 \\
\hline
\end{tabular}

Bold font indicates $P<0.05$.

${ }^{\mathrm{a} D}$ Data at $52 \mathrm{wks}$ were considered including patients still on treatment at that time point or patients who dropped out; patients with fewer than 52 weeks of observation but still on treatment were excluded.

Abbreviations: CD, Crohn's disease; UC, ulcerative colitis.

between continuous variables were assessed with the Mann-Whitney $U$ test. The long-term clinical effectiveness of VDZ beyond the first 52 weeks of treatment was evaluated with the Kaplan-Meier method.

GraphPad Prism version 8 (GraphPad Software, San Diego, CA, USA) was used to carry out statistical analyses. The presence of a statistical significance for $P$ was set at $<0.05$.

\section{3 | RESULTS}

\section{1 | Study population}

In total, 49 patients received VDZ during the study period: 21 with $U C$ and one with IBD-U whom we considered together as in the UC group for all analyses, and 27 with CD. Table 1 shows the base characteristics of the groups. The CD and UC groups were homogeneous except for their age at diagnosis (younger in $C D, P=0.006$ ) and disease duration (longer for $C D, P=0.01$ ). An additional dose at week 10 was given in 14 (52\%) patients with CD. Dose intensification was attempted in 15 (31\%) of all patients, with clinical benefit to six (40\%) of them.

\subsection{Clinical and endoscopic outcomes}

The main end-point results of the study are summarized in Table 2. At 14-week follow-up, clinical response was achieved by 23 (85\%) patients with CD and 11 (50\%) patients with UC, with a statistically significant better outcome in CD ( $P=0.01) ; 18(67 \%)$ patients with $C D$ and 7 (32\%) with UC were considered as being in clinical remission at the same time point $(P=0.03)$. Three patients with experience of anti-TNF (two with UC and one with CD) discontinued treatment before the end of induction regimen because of an inadequate response to severe disease and underwent surgery. At week 24,23

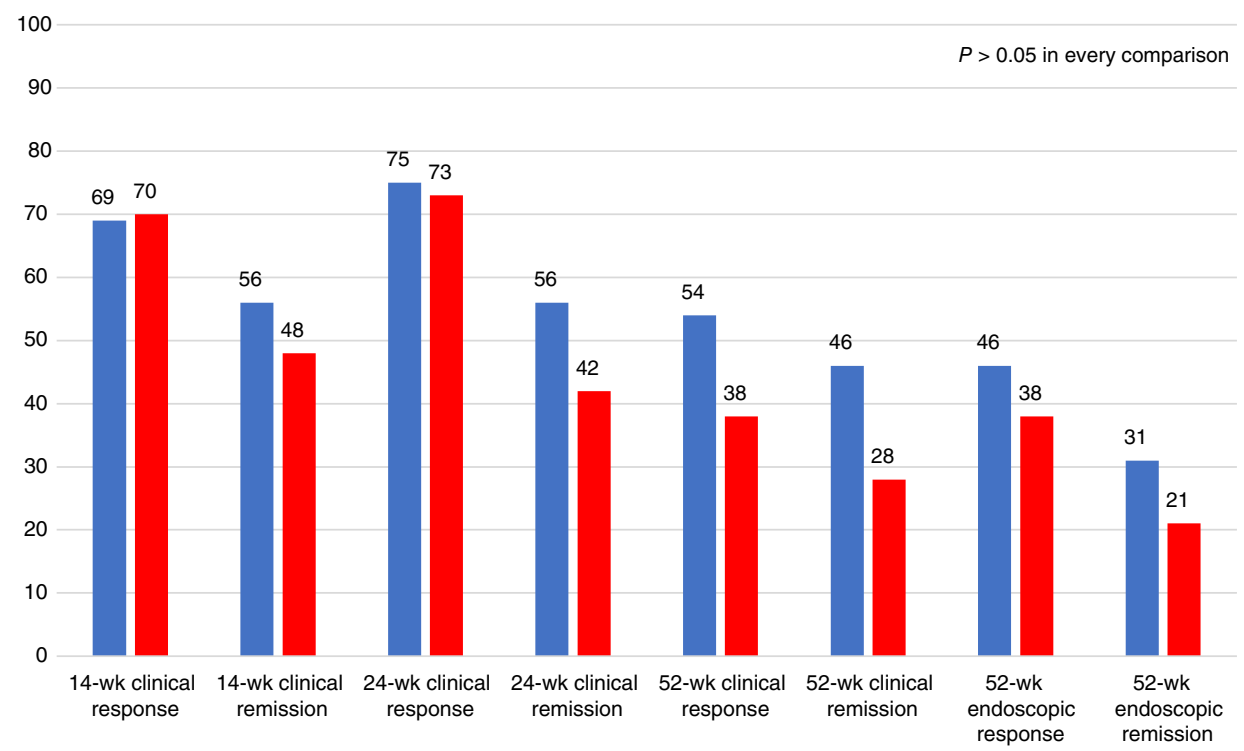

FIGURE 1 Clinical and endoscopic outcomes at the three time points: 14 , 24 , and 52 weeks. No statistically significant difference was found between the two groups of $\square$ biologicnaive and $\square$ biologic-experienced patients [Color figure can be viewed at wileyonlinelibrary.com] 
(85\%) patients with CD and 13 (59\%) with UC had a clinical response $(P>0.05)$; 16 (59\%) patients with CD and 7 (32\%) with UC achieved clinical remission $(P=0.04)$. At this observation point, two other patients prematurely stopped treatment due to loss of response.

At 52 weeks, seven patients had received VDZ for less than the selected time point, thus, they were excluded from data analysis and we considered only the patients who had undergone at least 52 weeks of treatment or who dropped out before reaching the time point because of inefficacy or adverse drug reactions. Therefore, 13 (59\%) patients with CD and 5 (25\%) with UC had a clinical response and 10 (45\%) with CD and 4 (20\%) with UC had clinical remission. An endoscopic response was found in 12 (54\%) CD patients and 5 (25\%) patients with UC, endoscopic remission in $6(27 \%)$ with CD and 4 (20\%) with UC. No statistical difference was noted between the two cohorts at this time point, either for clinical or for endoscopic outcomes. All patients with an endoscopic response were still receiving treatment at the end of the study (up to $28 \mathrm{mo}$ ). Median treatment duration was 52 weeks (IQR 36-64 weeks) in the overall cohort, and 76 weeks (IQR 56-88 weeks) in patients who continued VDZ for over 12 months. No statistically significant difference in terms of clinical and endoscopic outcomes was demonstrated between patients with previous exposure to anti-TNF drugs and naive patients, despite a trend of better responses in the naive group (Figure 1).

The CD and UC groups did not differ in terms of a steroid-free remission rate at 52 weeks, which was $41 \%(9 / 22)$ in those with CD and $20 \%(4 / 20)$ in those with UC $(P=0.19)$. CRP gradually decreased in patients with $C D$ and $U C$, with a drop of $0.51 \mathrm{mg} / \mathrm{dL}$ from baseline to 9 months. A lower baseline CRP was also a good response predictor at week $14(P=0.02)$.

\section{3 | Safety profile}

Mild to moderate adverse drug reactions were reported in $47 \%$ (23/49) of patients and none of them discontinued VDZ due to adverse drug reactions. The most frequent adverse drug reactions were upper respiratory $(n=13)$ and urinary $(n=4)$ infections; no safety concerns were reported. Concomitant therapies (immunosuppressants and corticosteroids) did not influence the adverse drug reaction rates.

\section{4 | Drug continuation}

Patients still on treatment after 52 weeks were followed up over time and data were collected and inserted in a Kaplan-Meier survival curve analysis. We reported comparable long-term drug survival in the two CD and UC groups $(P=0.08$, Figure 2). At 52 weeks, the overall probability of continuing VDZ treatment was $54 \%$ (39\% for UC, $67 \%$ for CD), while at 104 weeks was $30 \%$ (20\% for UC, $37 \%$ for CD).

\section{4 | DISCUSSION}

Real-world data on VDZ effectiveness and tolerability have started to accumulate in the last 2 years. Our single-center data collection is the

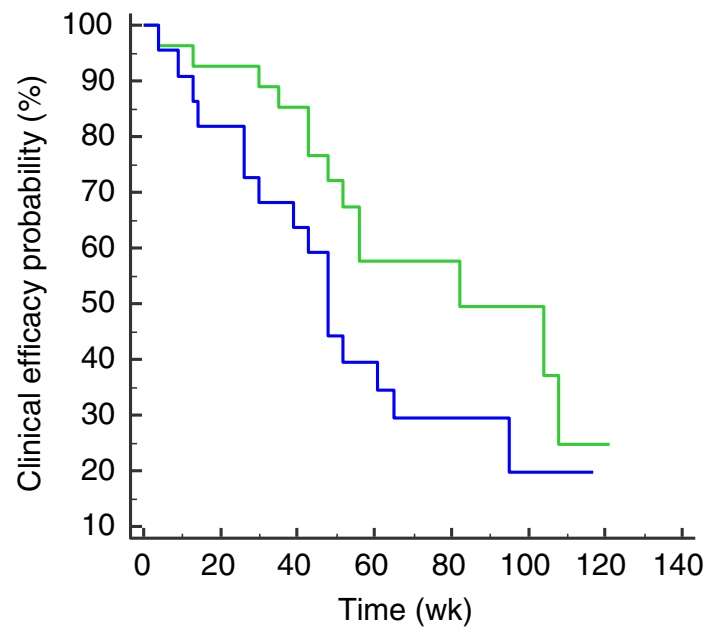

FIGURE 2 Kaplan-Meier survival curve analysis comparing the long-term clinical efficacy of vedolizumab in patients with ulcerative colitis and _C Crohn's disease (CD). Despite a trend towards higher efficacy in $C D$, no statistically significant difference was found $(P=0.08)$ [Color figure can be viewed at wileyonlinelibrary.com]

third evaluation after that of Kotze et $\mathrm{al}^{27}$ and the VICTORY Consortium studies ${ }^{20,31}$ to analyze both a long-term follow-up of at least 1 year and routine endoscopic assessment.

The GEMINI randomized clinical trial published in $2013^{5,7}$ led to the approval of VDZ for the treatment of patients with CD and UC, including both participants who were naive to anti-TNF agents and those who had experience of them. However, in this context of highly selective context it was to compare their findings with those of subsequent observational series. Therefore, it is prudent to discuss our data in the light of other real-life scenarios results.

In our analysis, VDZ was surprisingly found to induce a faster clinical remission in CD than in UC. The same results were shown by Kopylov et $\mathrm{al}^{12}$ with similar rates of response and remission both for CD and UC. Other authors ${ }^{17,18,23,28}$ found no difference in $C D$ and UC cohorts after the induction phase, while in one study a higher clinical benefit in patients with $U^{29}$ was seen, with a cumulative clinical remission plus response rate after 14 weeks of $91.2 \%$ in UC and $78.5 \%$ in $C D(P=0.02)$. Again, at 52 weeks the trend of the response in our study was favorable to $C D$ patients despite a lack of statistical significance; in other real-life studies, the two groups were comparable ${ }^{15}$ or there were higher response rates in $\mathrm{UC}^{27,29,30}$ In addition, despite the better clinical results in $\mathrm{CD}$, our endoscopic remissions at 52 weeks ( $15 \%$ UC and $27 \%$ CD) were very low compared with the other available data (41\% of UC and $63 \%$ of CD in the VICTORY studies $^{20,31}$ and $25.9 \%$ of CD and $47.8 \%$ of UC patients in a large Canadian cohort $^{27}$ ). A possible explanation for these findings may be the high percentage of patients with severe disease and patients with anti-TNF experience at baseline in our cohort.

As regards the steroid-free remission rate, our rates $(41 \% \mathrm{CD}$ and $20 \%$ UC) were similar to those of the VICTORY studies (34\% CD and $37 \%$ UC) and higher than the one reported by Stallmach et al (15\% for $\mathrm{CD}$ and $22 \%$ for $\mathrm{UC}) .{ }^{15}$ With respect to adverse drug reactions, the 
safety profile in our cohort was comparable to that described both in registrational trials and in other populations. ${ }^{41}$

Our Kaplan-Meier survival analysis reported lower response rates at 1 and 2 years than Kotze et $\mathrm{al}^{27}$ (56\% vs $70 \%$ at 52 weeks, $30 \%$ vs $53.2 \%$ at $104 \mathrm{wks}$ ), showing that the trend of sustained remission in patients with initial response is not to be expected in all clinical scenarios. In this regard, the perfect timing for defining a primary response or non-response to VDZ is currently unknown, as its effect may be slower than what is common in an anti-TNF response. ${ }^{42}$ Therefore, it appears advisable to wait for at least 14 weeks or more before discontinuation due to nonresponse; in fact, in our setting the earlier discontinuation of treatment occurred only in patients who had to be urgently referred to surgery.

Some studies have shown better treatment outcomes in biologicnaive patients. ${ }^{6,31,43}$ In our analysis, being naive or experienced with anti-TNF was not a significant predictor of response, and this has also been reported in other real-life settings. ${ }^{17,28}$

Recently, a score to predict VDZ response in patients with CD has been developed and the cohort from the VICTORY study has been used to validate the predictive score. ${ }^{44}$ Unluckily, baseline albumin was not available for most of our patients and the prediction score could not be used to explain the unexpectedly good clinical endpoints in our $\mathrm{CD}$ cohort.

The strong points of our study are the long period of evaluation and the routine endoscopic assessment that were available for all patients reaching 52 weeks of treatment or dropping out of the study. Most of the other real-world settings have the drawback of limited or no endoscopic results. Our study also had some limitations. First, it had a retrospective setting and this could have led to possible biases. For example, in the estimation of clinical and endoscopic activity, we partially overcame this problem by using standardized spreadsheets for clinical assessment and the agreement of two expert endoscopists for the endoscopic scores. Second, despite our detailed questionnaire, minor adverse events and infections might have been omitted by patients, resulting in a possible underestimation compared with clinical trials. Moreover, at the time of our analysis it was not possible to measure the VDZ trough level; consequently, dose intensification was based only on its clinical efficacy. In the future, the possibility of therapeutic drug monitoring will help the optimization of treatment and the more precise management of a loss of response. ${ }^{45}$

The place of VDZ in the biologic treatment algorithm is still debated and should be personalized. In fact, VDZ is to be preferred in patients who have positive history of opportunistic or serious infections, past malignancy, and elderly patients due to its attractive safety profile. ${ }^{46}$ A study has suggested a combination therapy of VDZ with anti-TNF, reporting no additional safety signals than that of single therapies on their own. ${ }^{47}$ Head-to-head trials versus anti-TNF agents are required to compare their safety and effectiveness.

\section{5 | CONCLUSION}

In conclusion, our study reported a high rate of clinical response in both the patients with UC and those with CD during the induction phase, as well as surprising better results in CD than in patients with UC. During the subsequent months of follow-up, our data found a higher drop out than the reported in the literatures, probably due to more severe disease at the baseline and an elevated percentage of biologic-experienced patients. As regards the safety profile, VDZ has been confirmed as a well-tolerated biologic that should be considered as a reliable first choice in patients with contraindication to anti-TNF agents.

In our center we believe it is very important to continue the follow-up of the patients still on treatment after 52 weeks to improve our understanding of the long-term efficacy and safety profile of VDZ. An additional systematic review and meta-analysis of the available data is required studying the clinical and endoscopic efficacy of VDZ at 1 year and beyond.

\section{ACKNOWLEDGEMENT}

We thank all the team of the Infusion Unit of our Gastroenterological Department for all their efforts that made this work possible.

\section{CONFLICT OF INTEREST}

The authors declare that they have no competing interests.

\section{ORCID}

Gabriele Dragoni (D) https://orcid.org/0000-0001-5752-5113

\section{REFERENCES}

1. Molander P, Sipponen $\mathrm{T}$, Kemppainen $\mathrm{H}$, et al. Achievement of deep remission during scheduled maintenance therapy with TNF $\alpha$-blocking agents in IBD. J Crohns Colitis. 2013;7(9):730-735.

2. Ma C, Huang V, Fedorak DK, et al. Crohn's disease outpatients treated with adalimumab have an earlier secondary loss of response and requirement for dose escalation compared to infliximab: a real life cohort study. J Crohns Colitis. 2014;8(11):1454-1463.

3. Qiu Y, Chen BL, Mao R, et al. Systematic review with meta-analysis: loss of response and requirement of anti-TNF $\alpha$ dose intensification in Crohn's disease. J Gastroenterol. 2017;52(5):535-554.

4. Jovani M, Danese S. Vedolizumab for the treatment of IBD: a selective therapeutic approach targeting pathogenic a4b7 cells. Curr Drug Targets. 2013;14(12):1433-1443.

5. Feagan BG, Rutgeerts P, Sands BE, et al. Vedolizumab as induction and maintenance therapy for ulcerative colitis. N Engl J Med. 2013; 369(8):699-710.

6. Sands BE, Sandborn WJ, Van Assche G, et al. Vedolizumab as induction and maintenance therapy for Crohn's disease in patients naïve to or who have failed tumor necrosis factor antagonist therapy. Inflamm Bowel Dis. 2017;23(1):97-106.

7. Sandborn WJ, Feagan BG, Rutgeerts $P$, et al. Vedolizumab as induction and maintenance therapy for Crohn's disease. $N$ Engl J Med. 2013;369(8):711-721.

8. Vermeire S, Loftus EV Jr, Colombel JF, et al. Long-term efficacy of vedolizumab for Crohn's disease. J Crohns Colitis. 2017;11(4):412-424.

9. Loftus EV Jr, Colombel JF, Feagan BG, et al. Long-term efficacy of vedolizumab for ulcerative colitis. J Crohns Colitis. 2017;11(4):400-411.

10. Sherman RE, Anderson SA, Dal Pan GJ, et al. Real-world evidence What is it and what can it tell us? N Engl J Med. 2016;375(23):22932297. 
11. Eriksson C, Marsal J, Bergemalm D, et al. Long-term effectiveness of vedolizumab in inflammatory bowel disease: a national study based on the Swedish National Quality Registry for Inflammatory Bowel Disease (SWIBREG). Scand J Gastroenterol. 2017;52(6-7): 722-729.

12. Kopylov U, Ron Y, Avni-Biron I, et al. Efficacy and safety of vedolizumab for induction of remission in inflammatory bowel disease-the Israeli real-world experience. Inflamm Bowel Dis 2017;23 (3):404-408.

13. Chaparro M, Sierra-Ausin M, Mesonero F, et al. Effectiveness and safety of vedolizumab for the induction of remission in inflammatory bowel disease patients. Unit Eur Gastroenterol J. 2016;2(Suppl):A264A265. https://www.ueg.eu/education/document/effectiveness-andsafety-of-vedolizumab-for-the-induction-of-remission-in-inflammatory-bowel-disease-patients/129907/.

14. Mankongpaisarnrung C, Mattar M, Charabaty A. Single-center experience: Vedolizumab in patients with Crohn's disease and ulcerative colitis at Georgetown University Hospital. Inflamm Bowel Dis. 2016;22 (Suppl):S32. https://doi.org/10.1097/01.MIB.0000480188.00054.c2.

15. Stallmach A, Langbein C, Atreya R, et al. Vedolizumab provides clinical benefit over 1 year in patients with active inflammatory bowel diseasea prospective multicenter observational study. Aliment Pharmacol Ther. 2016;44(11-12):1199-1212.

16. Vivio EE, Kanuri N, Gilbertsen JJ, et al. Vedolizumab effectiveness and safety over the first year of use in an IBD clinical practice. J Crohns Colitis. 2016;10(4):402-409.

17. Macaluso FS, Orlando R, Fries W, et al. The real-world effectiveness of vedolizumab on intestinal and articular outcomes in inflammatory bowel diseases. Dig Liver Dis. 2018;50(7):675-681.

18. Amiot A, Grimaud JC, Peyrin-Biroulet L, et al. Effectiveness and safety of vedolizumab induction therapy for patients with inflammatory bowel disease. Clin Gastroenterol Hepatol. 2016;14(11):15931601.e2.

19. De Vos M, Dhooghe B, Vermeire S, et al. Efficacy of vedolizumab for induction of clinical response and remission in patients with moderate to severe inflammatory bowel disease who failed at least two TNF antagonists. Unit Eur Gastroenterol J. 2018;6(3):439-445.

20. Dulai PS, Singh S, Jiang $X$, et al. The real-world effectiveness and safety of vedolizumab for moderate-severe Crohn's disease: results from the US VICTORY consortium. Am J Gastroenterol. 2016;111(8): 1147-1155.

21. Williet N, Boschetti G, Fovet M, et al. Association between low trough levels of Vedolizumab during induction therapy for inflammatory bowel diseases and need for additional doses within 6 months. Clin Gastroenterol Hepatol. 2017;15(11):1750-1757.e3.

22. Samaan MA, Pavlidis P, Johnston E, et al. Vedolizumab: early experience and medium-term outcomes from two UK tertiary IBD centres. Frontline Gastroenterol. 2017;8(3):196-202.

23. Baumgart DC, Bokemeyer B, Drabik A, Stallmach A, Schreiber S; Vedolizumab Germany Consortium. Vedolizumab induction therapy for inflammatory bowel disease in clinical practice-a nationwide consecutive German cohort study. Aliment Pharmacol Ther. 2016;43(10): 1090-1102.

24. Iborra M, Maroto N, Navarro-Cortes $\mathrm{P}$, et al. Vedolizumab, an adequate option in medically refractory and thiopurine-intolerant inflammatory bowel disease patients. J Crohns Colitis. 2018;12(Suppl): S472-S473.

25. Allegretti JR, Barnes EL, Stevens B, et al. Predictors of clinical response and remission at 1 year among a multicenter cohort of patients with inflammatory bowel disease treated with vedolizumab. Dig Dis Sci. 2017;62(6):1590-1596.

26. Hoffmann P, Krisam J, Stremmel W, Gauss A. Real-world outcomes of vedolizumab therapy in ulcerative colitis and Crohn's disease at a tertiary referral center. Dig Dis. 2019;37(1):33-44.
27. Kotze PG, Ma C, Almutairdi A, et al. Real-world clinical, endoscopic and radiographic efficacy of vedolizumab for the treatment of inflammatory bowel disease. Aliment Pharmacol Ther. 2018;48(6):626-637.

28. Ylisaukko-oja T, Aaltonen J, Nuutinen $\mathrm{H}$, et al. High treatment persistence rate and significant endoscopic healing among real-life patients treated with vedolizumab-a Finnish nationwide inflammatory bowel disease cohort study (FINVEDO). Scand J Gastroenterol. 2018;53(2): 158-167.

29. Lenti MV, Levison S, Eliadou E, et al. A real-world, long-term experience on effectiveness and safety of vedolizumab in adult patients with inflammatory bowel disease: the Cross Pennine study. Dig Liver Dis. 2018;50(12):1299-1304.

30. Chaparro M, Garre A, Ricart E, et al. Short and long-term effectiveness and safety of vedolizumab in inflammatory bowel disease: results from the ENEIDA registry. Aliment Pharmacol Ther. 2018;48 (8):839-851.

31. Narula N, Peerani F, Meserve J, et al. Vedolizumab for ulcerative colitis: treatment outcomes from the VICTORY consortium. Am J Gastroenterol. 2018;113(9):1345. https://doi.org/10.1038/ s41395-018-0162-0.

32. Magro F, Gionchetti P, Eliakim R, et al. Third European evidencebased consensus on diagnosis and management of ulcerative colitis. Part 1: definitions, diagnosis, extra-intestinal manifestations, pregnancy, cancer surveillance, surgery, and ileo-anal pouch disorders. J Crohns Colitis. 2017;11(6):649-670.

33. Gomollón F, Dignass A, Annese V, et al. 3rd European evidence-based consensus on the diagnosis and management of Crohn's disease 2016: part 1: diagnosis and medical management. J Crohns Colitis. 2017;11(1):3-25.

34. Rahier JF, Magro F, Abreu C, et al. Second European evidence-based consensus on the prevention, diagnosis and management of opportunistic infections in inflammatory bowel disease. J Crohns Colitis. 2014; 8(6):443-468.

35. Lewis JD, Chuai S, Nessel L, Lichtenstein GR, Aberra FN, Ellenberg JH. Use of the noninvasive components of the Mayo score to assess clinical response in ulcerative colitis. Inflamm Bowel Dis. 2008;14(12):1660-1666.

36. Harvey RF, Bradshaw JM. A simple index of Crohn's-disease activity. Lancet. 1980;315(8167):514.

37. Daperno M, D'Haens G, Van Assche G, et al. Development and validation of a new, simplified endoscopic activity score for Crohn's disease: the SES-CD. Gastrointest Endosc. 2004;60(4):505-512.

38. Rutgeerts P, Geboes K, Vantrappen G, Beyls J, Kerremans R, Hiele M. Predictability of the postoperative course of Crohn's disease. Gastroenterology. 1990;99(4):956-963.

39. Rutgeerts P, Sandborn WJ, Feagan BG, et al. Infliximab for induction and maintenance therapy for ulcerative colitis. N Engl J Med. 2005; 353(23):2462-2476.

40. Charlson ME, Pompei P, Ales KL, MacKenzie CR. A new method of classifying prognostic comorbidity in longitudinal studies: development and validation. J Chronic Dis. 1987;40(5):373-383.

41. Colombel JF, Sands BE, Rutgeerts $P$, et al. The safety of vedolizumab for ulcerative colitis and Crohn's disease. Gut. 2017;66(5):839-851.

42. Engel T, Ungar B, Yung DE, Ben-Horin S, Eliakim R, Kopylov U. Vedolizumab in IBD-lessons from real-world experience; a systematic review and pooled analysis. J Crohns Colitis. 2018;12(2):245-257.

43. Feagan BG, Rubin DT, Danese S, et al. Efficacy of vedolizumab induction and maintenance therapy in patients with ulcerative colitis, regardless of prior exposure to tumor necrosis factor antagonists. Clin Gastroenterol Hepatol. 2017;15(2):229-239.e5.

44. Dulai PS, Boland BS, Singh S, et al. Development and validation of a scoring system to predict outcomes of vedolizumab treatment in patients with Crohn's disease. Gastroenterology. 2018;155(3):687695.e10. 
45. Shmidt E, Kochhar G, Hartke J, et al. Predictors and management of loss of response to Vedolizumab in inflammatory bowel disease. Inflamm Bowel Dis. 2018;24(11):2461-2467.

46. Pouillon L, Van Stappen J, Bossuyt P, Danese S, Peyrin-Biroulet L. Should we use anti-tumor necrosis factor agents or vedolizumab as first-line biological therapy in ulcerative colitis? Best Pract Res Clin Gastroenterol. 2018;32-33:17-25.

47. Ben-Horin S, Ungar B, Kopylov U, et al. Safety, efficacy and pharmacokinetics of vedolizumab in patients with simultaneous exposure to an anti-tumour necrosis factor. Aliment Pharmacol Ther. 2018;47(8): 1117-1125.
How to cite this article: Dragoni G, Bagnoli S, Le Grazie M, et al. Long-term efficacy and safety of vedolizumab in patients with inflammatory bowel diseases: A real-life experience from a tertiary referral center. J Dig Dis. 2019;20:235-242. https:// doi.org/10.1111/1751-2980.12748 